\title{
Gohberg-Semencul type formula and application for the inverse of a conjugate-Toeplitz matrix involving imaginary circulant matrices
}

\author{
Xiaoyu Jiang, Kicheon Hong* \\ Dept. of Information and Telecommunications Engineering, The University of Suwon, Wau-ri, Bongdam-eup, Hwaseong-si, \\ Gyeonggi-do, 445-743, Korea.
}

Communicated by M. De La Sen

\begin{abstract}
Gohberg-Semencul type inverse formula of conjugate-Toeplitz (CT) is obtained by constructing a kind of imaginary cyclic displacement transform. The stability of decomposition formula of inverse is investigated, and its algorithm is also given. Numerical example is provided to verify the feasibility of the inverse formula. How the analogue of our formula leads to a more efficient way to solve the conjugate-Toeplitz linear system of equations is proposed. The corresponding inverse, stability, and algorithm of conjugate-Hankel (CH) matrix are also considered. (C)2017 All rights reserved.
\end{abstract}

Keywords: Conjugate-Toeplitz matrix, conjugate-Hankel matrix, stability, imaginary cyclic displacement, fast Fourier transform.

2010 MSC: 15A09, 15A15, 15A69, 65F05.

\section{Introduction}

Toeplitz matrix has become a satisfactory tool in restoration of signals and images [5, 26, 27]. In [4, 7], the authors introduced a generalization of Toeplitz matrices, called conjugate-Toeplitz (CT) matrices, and showed that certain properties of Toeplitz matrices could be extended to CT matrices. The explicit inverse of nonsingular conjugate-Toeplitz and conjugate-Hankel matrices are provided [12]. The inverses of conjugate-Toeplitz (CT) and conjugate-Hankel $(\mathrm{CH})$ matrices can be expressed by the Gohberg-Semencul type formula [17]. Gover and Barnett [8] introduced a corresponding algorithm for any strongly nonsingular CT matrix (i.e., all of its leading principal minors are nonzero). Algorithms for inverting CT matrices and solving CT systems of equations, using $\mathrm{O}\left(\mathrm{n}^{2}\right)$ flops for matrices of order $n$, were given. The purpose of [9] is to show that some known properties of Toeplitz and CT matrices can be extended to rT matrices. In [22], an expression of the inverse of a conjugate Toeplitz matrix is obtained. The necessary conditions of applying the generalized Trench algorithm for CT matrices are discussed. It is shown that there exist strongly invertible CT matrices to which the algorithm may not be applied.

Gohberg-Semencul type formula for inverses of conjugate-Toeplitz and conjugate-Hankel matrix only mentioned in $[4,12,17]$ have not been fully exploited.

\footnotetext{
*Corresponding author

Email addresses: jxy19890422@sina.com (Xiaoyu Jiang), Kchong@suwon.ac.kr (Kicheon Hong)
} 
Let $T=\left[t_{j-k}\right]_{j, k=0}^{n-1}$ be a real symmetric positive-definite Toeplitz matrix of order $n$. There are several well-known $\mathrm{O}\left(\mathrm{n}^{2}\right)$ algorithms for solving the linear system of equations $T x=b$, and more recently, several $\mathrm{O}\left(n \log ^{2} n\right)$ algorithms have been developed. See, for example, $[2,3,11,29]$ and the references contained therein. Algorithms from both of these classes often rely, either implicitly or explicitly, on the Gohberg-Semencul formula [6], which provides a decomposition of $\mathrm{T}^{-1}$ into the sum of products of lower triangular and upper triangular Toeplitz matrices. Although we will consider $T$ to be a real positive-definite Toeplitz matrix, formula presented by Gohberg and Semencul apply to the inverse of any invertible Toeplitz matrix.

In [18], the authors presented an innovative patterned matrix, RFPL-Toeplitz matrix, is neither the extension of Toeplitz matrix nor its special case. The group inverse of this new patterned matrix can be represented as the sum of products of lower and upper triangular Toeplitz matrices, then the explicit expression and the decomposition of the group inverse is given. The inverses of CUPL-Toeplitz and CUPL-Hankel matrices can be expressed by the Gohberg-Heinig type formula in [13]. Jiang and Hong [15] derived the formulas on representation of the inverses of the CUPL Toeplitz matrices in the form of sums of products of factor $(1,1)$-circulants and $(-1,-1)$-circulants. The stability of the algorithms emerging from Toeplitz matrix inversion formulas is considered in [10, 30]. Xie and Wei [31] presented a stability analysis of Gohberg-Semencul-Trench type for Moore-Penrose and group inverses of Toeplitz matrices. Toeplitz inversion formula involving circulant matrices have also been presented in [1, 23, 24].

As far as we know, there is no more efficient algorithms for solving the linear system of equations $\mathrm{T}_{\mathrm{C}} \mathrm{x}=\mathrm{b}$ and $\mathrm{H}_{\mathrm{C}} \mathrm{x}=\mathrm{b}$. We hope that this paper will help in changing this to provide an algorithm for the effective application.

We remark that imaginary cyclic displacement structure plays a critical role in finding the inverse of CT matrix. At present, the articles about imaginary circulant matrix are very few, some interrelated basic knowledge is stated only in [14, 21, 25, 28].

We provide CT matrix inversion formula as a sum of two products of imaginary circulant matrix and upper triangular matrices. It will be shown the number of real arithmetic operations is less than the known results to solve the conjugate-Toeplitz and conjugate-Hankel linear system of equations.

In this paper, $\mathbb{R}$ denotes the set of real numbers and $i=\sqrt{-1}$. Now allow us to introduce some basic knowledges about $\mathrm{CT}$ and $\mathrm{CH}$ matrices, which are stated in $[4,7]$.

Definition 1.1. Let $c(x)=\bar{x}$ denote the complex conjugate of $x$. In particular note that $c^{2 m}(x)=x$, and $c^{2 m-1}(x)=\bar{x}$, for all positive integers $m$.

Definition 1.2. An $n \times n$ matrix $T_{C}=\left(t_{j k}\right)$ is conjugate-Toeplitz (CT) if $t_{j+1, k+1}=c\left(t_{j k}\right)$ for all $j, k$, that is,

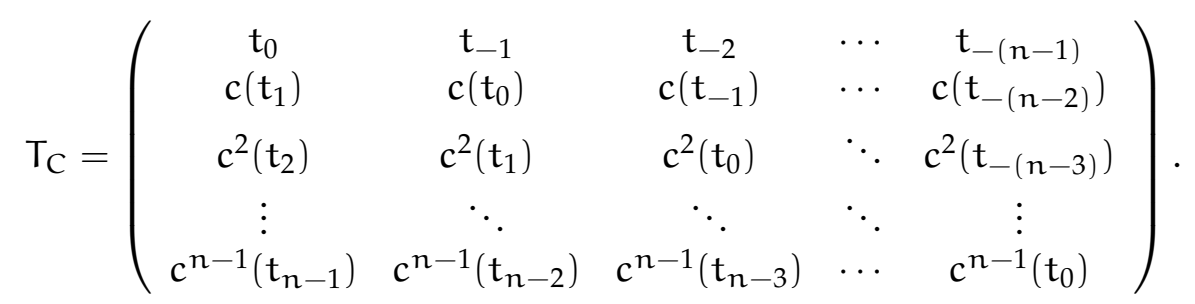

Definition 1.3. An $n \times n$ matrix $H_{C}=\left(h_{j k}\right)$ is conjugate-Hankel $(C H)$ if $h_{j+1, k}=c\left(h_{j, k+1}\right)$ for all $j$, $k$, that is,

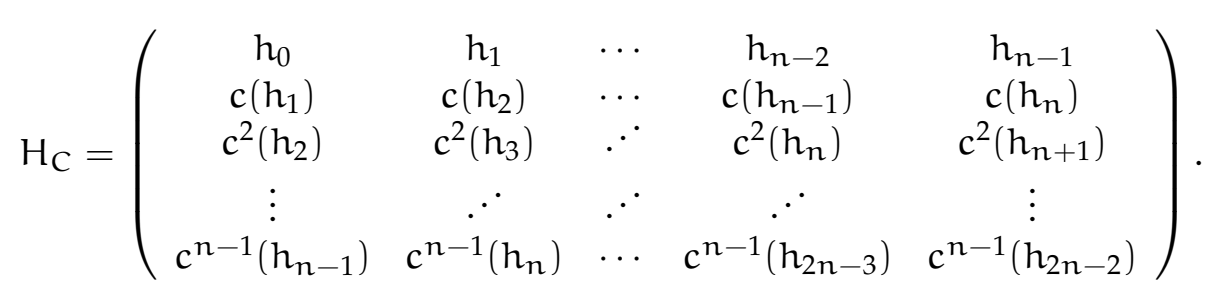




\section{Conjugate-Toeplitz inversion formula}

Constructing a kind of imaginary cyclic displacement, we propose inversion formula for CT matrix as a sum of two products of imaginary circulant and upper triangular matrices.

Lemma 2.1. Let $\mathrm{T}_{\mathrm{C}}=\left(\mathrm{t}_{\mathrm{jk}}\right)$ be an $\mathrm{n} \times \mathrm{n}$ conjugate-Toeplitz matrix defined in equation (1.1) with $\mathrm{T}_{\mathrm{C}}$ invertible and $t_{j k} \in i \mathbb{R}$. Then $T_{C}$ satisfies the formula

$$
\Pi \mathrm{T}_{\mathrm{C}}+\mathrm{T}_{\mathrm{C}} \Pi=v e_{\mathrm{n}}^{\top}-e_{1} v^{\top} \widehat{\mathrm{J}},
$$

where

$$
\begin{aligned}
\Pi=\left(\begin{array}{cccc}
0 & \cdots & 0 & i \\
1 & \ddots & & 0 \\
& \ddots & \ddots & \vdots \\
& & 1 & 0
\end{array}\right), & \widehat{J}=\left(\begin{array}{c}
(-1)^{0} \\
(-1)^{n-1} \\
e_{1}=\left(\begin{array}{c}
0 \\
0 \\
\vdots \\
0
\end{array}\right), e_{n}=\left(\begin{array}{c}
0 \\
c^{0}\left(t_{1-n}-i t_{1}\right) \\
\vdots \\
1
\end{array}\right), \\
c^{n-3}\left(t_{-2}-i t_{n-2}\right) \\
c^{n-2}\left(t_{-1}-i t_{n-1}\right)
\end{array}\right) .
\end{aligned}
$$

We do not give a detail proof for Lemma 2.1, its technical skill can be calculated directly.

Theorem 2.2. Let $\mathrm{T}_{\mathrm{C}}=\left(\mathrm{t}_{\mathrm{j}}\right)$ be an $\mathrm{n} \times \mathrm{n}$ conjugate-Toeplitz matrix defined with $\mathrm{T}_{\mathrm{C}}$ invertible and $\mathrm{t}_{\mathrm{jk}} \in \mathrm{i} \mathbb{R}$. If each of the systems of equations $\mathrm{T}_{\mathrm{C}} \mathrm{x}=v, \mathrm{~T}_{\mathrm{C}} \mathrm{y}=\mathrm{e}_{1}$ are solvable,

$$
x=\left(\begin{array}{llll}
x_{1} & x_{2} & \cdots & x_{n}
\end{array}\right)^{\top}, y=\left(\begin{array}{llll}
y_{1} & y_{2} & \cdots & y_{n}
\end{array}\right)^{\top},
$$

then $\mathrm{T}_{\mathrm{C}}$ is invertible and

$$
\mathrm{T}_{\mathrm{C}}^{-1}=\mathbb{I}_{1}^{\top} \mathrm{U}_{1}+\mathbb{I}_{2}^{\top} \mathrm{U}_{2}
$$

where

$$
\begin{aligned}
& \mathbb{I}_{1}=\left(\begin{array}{cccc}
y_{1} & y_{2} & \cdots & y_{n} \\
i y_{n} & y_{1} & \ddots & \vdots \\
\vdots & \ddots & \ddots & y_{2} \\
i y_{2} & \cdots & i y_{n} & y_{1}
\end{array}\right), \quad \quad \mathbb{I}_{2}=\left(\begin{array}{cccc}
x_{1} & x_{2} & \cdots & x_{n} \\
i x_{n} & x_{1} & \ddots & \vdots \\
\vdots & \ddots & \ddots & x_{2} \\
i x_{2} & \cdots & i x_{n} & x_{1}
\end{array}\right), \\
& \mathrm{U}_{1}=\left(\begin{array}{cccc}
(-1)^{0} & (-1)^{1} x_{n} & \cdots & (-1)^{n-1} x_{2} \\
& (-1)^{1} & \cdots & (-1)^{n-1} x_{3} \\
& & \ddots & \vdots \\
& & \ddots & (-1)^{n-1} x_{n}
\end{array}\right), \quad \mathrm{U}_{2}=\left(\begin{array}{ccccc}
0 & (-1)^{2} y_{n} & (-1)^{3} y_{n-1} & \cdots & (-1)^{n} y_{2} \\
& & (-1)^{3} y_{n} & \ddots & \vdots \\
& & & \ddots & (-1)^{n} y_{n-1} \\
& & & \ddots & (-1)^{n} y_{n}
\end{array}\right) \text {, }
\end{aligned}
$$

and $\mathbb{I}_{1}, \mathbb{I}_{2}$ are both imaginary circulant matrices [14, 16, 19-21, 25].

Proof. From equation (2.1) and $\mathrm{T}_{C} x=v, \mathrm{~T}_{C} y=e_{1}$, we have

$$
\Pi \mathrm{T}_{\mathrm{C}}=-\mathrm{T}_{\mathrm{C}} \Pi+v e_{\mathrm{n}}^{\top}-e_{1} v^{\top} \widehat{\mathrm{J}}=\mathrm{T}_{\mathrm{C}}\left(-\Pi+x e_{\mathrm{n}}^{\top}-\mathrm{y} v^{\top} \widehat{\mathrm{J}}\right) .
$$


Then

$$
\Pi^{j} T_{C}=\Pi^{j-1} T_{C}\left(-\Pi+x e_{n}^{\top}-y v^{\top} \widehat{J}\right)=T_{C}\left(-\Pi+x e_{n}^{\top}-y v^{\top} \widehat{J}\right)^{j} .
$$

Therefore,

$$
\Pi^{j} e_{1}=\Pi^{j} T_{C} y=T_{C}\left(-\Pi+x e_{n}^{\top}-y v^{\top} \widehat{J}\right)^{j} y
$$

Let

$$
\mu_{j}=\left(-\Pi+x e_{n}^{\top}-y v^{\top} \widehat{J}^{j-1} y \text { and } \hat{T}_{C}=\left(\begin{array}{llll}
\mu_{1} & \mu_{2} & \cdots & \mu_{n}
\end{array}\right) .\right.
$$

Then

$$
\begin{aligned}
& \mathrm{T}_{C} \mu_{j}=\mathrm{T}_{C}\left(-\Pi+x e_{n}^{\top}-y v^{\top} \widehat{J}\right)^{j-1} y=\Pi^{j-1} e_{1}=e_{j}, \\
& \mathrm{~T}_{C} \hat{\top}_{C}=\mathrm{T}_{C}\left(\begin{array}{llll}
\mu_{1} & \mu_{2} & \cdots & \mu_{n}
\end{array}\right)=\left(\begin{array}{llll}
e_{1} & e_{2} & \cdots & e_{n}
\end{array}\right)=I_{n},
\end{aligned}
$$

where $I_{n}$ denotes the $n \times n$ identity matrix. So matrix $T_{C}$ is invertible, and $T_{C}^{-1}$ is $\hat{T}_{C}$. It is easy to get

$$
\begin{aligned}
& \mu_{1}=y, \quad \mu_{j}=\left(-\Pi+x e_{n}^{\top}-y v^{\top} \widehat{J}\right) \mu_{j-1}(j=1,2, \cdots, n), \\
& \mu_{j}=T_{C}^{-1} e_{j}, \widehat{J} e_{j}=(-1)^{j} e_{n-j+1}, \widehat{J} T_{C} \widehat{J}=T_{C}^{T}, \widehat{J}^{-1}=(-1)^{n+1} \widehat{J} .
\end{aligned}
$$

Then, for $j>1$,

$$
\begin{aligned}
\mu_{j} & =-\Pi \mu_{j-1}+x e_{n}^{\top} \mu_{j-1}-y v^{\top} \widehat{J} \mu_{j-1} \\
& =-\Pi \mu_{j-1}+x e_{n}^{\top} T_{C}^{-1} e_{j-1}-y v^{\top} \widehat{J}_{C}^{-1} e_{j-1} \\
& =-\Pi \mu_{j-1}+x e_{n}^{\top} \widehat{J}^{-1} \widehat{J}_{C}^{-1} \widehat{J J}^{-1} e_{j-1}-y v^{\top} \widehat{J}_{C}^{-1} \widehat{J J}^{-1} e_{j-1} \\
& =-\Pi \mu_{j-1}+x e_{1}^{\top}\left(T_{C}^{-1}\right)^{\top}(-1)^{j} e_{n-j+2}-y v^{\top}\left(T_{C}^{-1}\right)^{\top}(-1)^{j} e_{n-j+2} \\
& =-\Pi \mu_{j-1}+(-1)^{j} x y^{\top} e_{n-j+2}-(-1)^{j} y x^{\top} e_{n-j+2} \\
& =-\Pi \mu_{j-1}+(-1)^{j} y_{n-j+2} x+(-1)^{j+1} x_{n-j+2} y .
\end{aligned}
$$

According to $\mu_{1}=y$ and equation (2.3), we have

$$
\begin{aligned}
& \mathrm{T}_{\mathrm{C}}^{-1}=\left(\begin{array}{llll}
\mu_{1} & \mu_{2} & \cdots & \mu_{\mathrm{n}}
\end{array}\right) \\
& =\left(\begin{array}{llll}
y & (-1)^{1} \Pi y & \cdots & (-1)^{n-1} \Pi^{n-1} y
\end{array}\right) \Psi_{1}+\left(\begin{array}{llll}
x & (-1)^{1} \Pi x & \cdots & (-1)^{n-1} \Pi^{n-1} x
\end{array}\right) \Psi_{2} \\
& =\left(\begin{array}{llll}
y & \Pi y & \cdots & \Pi^{n-1} y
\end{array}\right) \mathbb{D} \Psi_{1}+\left(\begin{array}{llll}
x & \Pi x & \cdots & \Pi^{n-1} x
\end{array}\right) \mathbb{D} \Psi_{2} \\
& =\left(\begin{array}{llll}
y & \Pi y & \cdots & \Pi^{n-1} y
\end{array}\right) \mathrm{U}_{1}+\left(\begin{array}{llll}
x & \Pi x & \cdots & \Pi^{n-1} x
\end{array}\right) \mathrm{U}_{2} \\
& =\left(\begin{array}{cccc}
y_{1} & i y_{n} & \cdots & i y_{2} \\
y_{2} & y_{1} & \ddots & \vdots \\
\vdots & \ddots & \ddots & i y_{n} \\
y_{n} & \cdots & y_{2} & y_{1}
\end{array}\right) \mathrm{U}_{1}+\left(\begin{array}{cccc}
x_{1} & i x_{n} & \cdots & i x_{2} \\
x_{2} & x_{1} & \ddots & \vdots \\
\vdots & \ddots & \ddots & i x_{n} \\
x_{n} & \cdots & x_{2} & x_{1}
\end{array}\right) \mathrm{U}_{2} \\
& =\mathbb{I}_{1}^{\mathrm{T}} \mathrm{U}_{1}+\mathbb{I}_{2}^{\mathrm{T}} \mathrm{U}_{2}
\end{aligned}
$$

where

$$
\Psi_{1}=\left(\begin{array}{cccc}
1 & (-1)^{1} x_{n} & \cdots & (-1)^{n-1} x_{2} \\
& 1 & \ddots & \vdots \\
& & \ddots & (-1)^{1} x_{n} \\
& & & 1
\end{array}\right), \Psi_{2}=\left(\begin{array}{ccccc}
0 & (-1)^{2} y_{n} & (-1)^{3} y_{n-1} & \cdots & (-1)^{n} y_{2} \\
& 0 & (-1)^{2} y_{n} & \ddots & \vdots \\
& & 0 & \ddots & (-1)^{3} y_{n-1} \\
& & & \ddots & (-1)^{2} y_{n}
\end{array}\right)
$$




$$
\mathbb{D}=\left(\begin{array}{cccc}
(-1)^{0} & & & \\
& (-1)^{1} & & \\
& & \ddots & \\
& & & (-1)^{n-1}
\end{array}\right)
$$

We remark that the formulas in the theorem are expressed by solving the systems of equations $T_{C} x=$ $v, \mathrm{~T}_{\mathrm{C}} \mathrm{y}=\mathrm{e}_{1}$. This provides an algorithm to compute the inverse of $\mathrm{T}_{\mathrm{C}}$.

\section{Conjugate-Hankel inversion formula}

In this section we provide inversion formula for $\mathrm{CH}$ matrix as a sum of two products of left imaginary circulant matrix and upper triangular matrices.

Lemma 3.1. Let $\mathrm{H}_{\mathrm{C}}=\left(\mathrm{h}_{\mathrm{jk}}\right)$ be an $\mathrm{n} \times \mathrm{n}$ conjugate-Hankel matrix with $\mathrm{H}_{\mathrm{C}}$ invertible and $\mathrm{h}_{\mathrm{jk}} \in i \mathbb{R}$. Then $\mathrm{H}_{\mathrm{C}}$ satisfies

$$
\Pi \mathrm{H}_{\mathrm{C}}+\mathrm{H}_{\mathrm{C}} \Pi^{\top}=\hat{v} e_{1}^{\top}-e_{1} \hat{v}^{\top} \mathbb{D},
$$

where $\Pi, \mathbb{D}$, and $e_{1}$ are defined above and

$$
\hat{v}=\left(\begin{array}{c}
0 \\
c^{0}\left(h_{0}-i h_{n}\right) \\
c^{1}\left(h_{1}-i h_{n+1}\right) \\
\vdots \\
c^{n-2}\left(h_{n-2}-i h_{2 n-2}\right)
\end{array}\right) .
$$

Theorem 3.2. Let $\mathrm{H}_{\mathrm{C}}=\left(\mathrm{h}_{\mathrm{jk}}\right)$ be a conjugate-Hankel matrix with $\mathrm{H}_{\mathrm{C}}$ invertible and $\mathrm{h}_{\mathrm{jk}} \in i \mathbb{R}$. If each of the systems of equations $\mathrm{H}_{\mathrm{C}} \xi=\hat{v}, \mathrm{H}_{\mathrm{C}} \eta=e_{1}$ are solvable, $\xi=\left(\begin{array}{llll}\xi_{1} & \xi_{2} & \cdots & \xi_{\mathrm{n}}\end{array}\right)^{\top}, \eta=\left(\begin{array}{llll}\eta_{1} & \eta_{2} & \cdots & \eta_{n}\end{array}\right)^{\top}$, then $\mathrm{H}_{\mathrm{C}}$ is invertible and

$$
\mathrm{H}_{\mathrm{C}}^{-1}=\mathbb{L}_{1} \Phi_{1}+\mathbb{L}_{2} \Phi_{2}
$$

where

$$
\begin{aligned}
& \mathbb{L}_{1}=\left(\begin{array}{ccccc}
\eta_{1} & \eta_{2} & \eta_{3} & \cdots & \eta_{n} \\
\eta_{2} & \eta_{3} & \cdots & \eta_{n} & i \eta_{1} \\
\eta_{3} & & . & i \eta_{1} & i \eta_{2} \\
\vdots & . & . & . \cdot & \vdots \\
\eta_{n} & i \eta_{1} & i \eta_{2} & \cdots & i \eta_{n-1}
\end{array}\right), \quad \quad \mathbb{L}_{2}=\left(\begin{array}{ccccc}
\xi_{1} & \xi_{2} & \xi_{3} & \cdots & \xi_{n} \\
\xi_{2} & \xi_{3} & \cdots & \xi_{n} & i \xi_{1} \\
\xi_{3} & & . & i \xi_{1} & i \xi_{2} \\
\vdots & . & . & . & \vdots \\
\xi_{n} & i \xi_{1} & i \xi_{2} & \cdots & i \xi_{n-1}
\end{array}\right),
\end{aligned}
$$

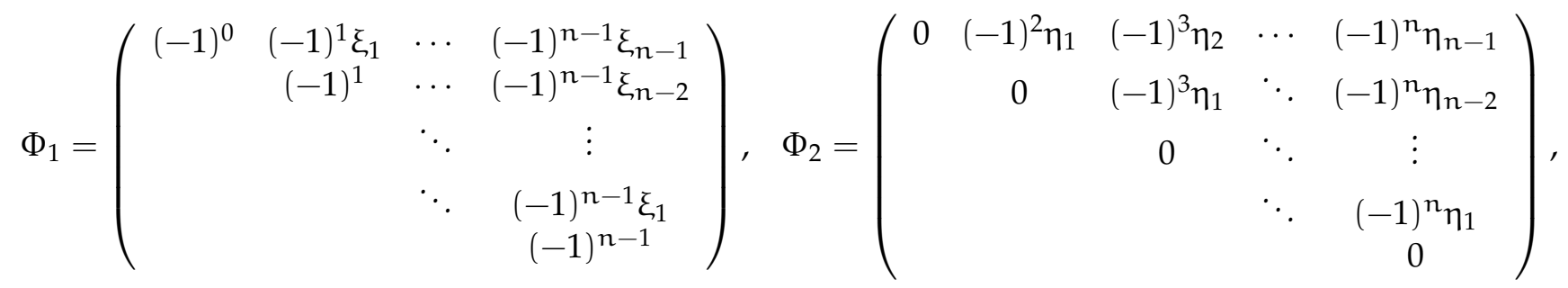

and $\mathbb{L}_{1}, \mathbb{L}_{2}$ are both left imaginary circulant matrices [21].

Proof. The proof is similar to Theorem 2.2. 


\section{Stability analysis of decomposition formulas (2.2) and (3.1)}

In this section, we will analyze the stability of the inversion formulas given in Theorems 2.2 and 3.2. Now we show the error analysis of the explicit inversion formulas for CT matrices and $\mathrm{CH}$ matrices in terms of the 1-norm, $\infty$-norm, and 2-norm, respectively. Denote

$$
\begin{array}{lll}
x=\left(x_{1}, x_{2} \ldots, x_{n-1}, x_{n}\right)^{\top}, & \bar{x}=\left(i x_{n} \ldots, i x_{3}, i x_{2}\right)^{\top}, \\
y=\left(y_{1}, y_{2} \ldots, y_{n-1}, y_{n}\right)^{\top}, & \bar{y}=\left(i y_{n} \ldots, i y_{3}, i y_{2}\right)^{\top}, \\
\xi=\left(\xi_{1}, \xi_{2} \ldots, \xi_{n-1}, \xi_{n}\right)^{\top}, & \bar{\xi}=\left(i \xi_{1} \ldots, i \xi_{n-2}, i \xi_{n-1}\right)^{\top}, \\
\eta=\left(\eta_{1}, \eta_{2} \ldots, \eta_{n-1}, \eta_{n}\right)^{\top}, & \bar{\eta}=\left(i \eta_{1} \ldots, i \eta_{n-2}, i \eta_{n-1}\right)^{\top} .
\end{array}
$$

Theorem 4.1. Let $\mathrm{T}_{\mathrm{C}}$ be an $\mathrm{n} \times \mathrm{n}$ conjugate-Toeplitz matrix and well conditioned. Let $\sigma>0$ and let $\hat{x}, \hat{\mathrm{y}}, \hat{\bar{x}}, \hat{\overline{\mathrm{y}}}$ be the corresponding numerical least squares solutions of the linear systems for deriving the formula (2.2). Denote by $\hat{\mathrm{T}}_{\mathrm{C}}^{-1}$ the inverse of $\hat{\mathrm{T}}_{\mathrm{C}}$. If $\frac{\|\hat{\mathrm{x}}-\mathrm{x}\|_{1}}{\|\mathrm{x}\|_{1}} \leqslant \sigma, \frac{\|\hat{\mathrm{y}}-\mathrm{y}\|_{1}}{\|y\|_{1}} \leqslant \sigma, \frac{\|\hat{\mathrm{x}}-\overline{\mathrm{x}}\|_{1}}{\|\overline{\mathrm{x}}\|_{1}} \leqslant \sigma, \frac{\|\hat{\mathrm{y}}-\overline{\mathrm{y}}\|_{1}}{\|\bar{y}\|_{1}} \leqslant \sigma$, then,

$$
\left\|\mathrm{T}_{\mathrm{C}}^{-1}-\hat{\mathrm{T}}_{\mathrm{C}}^{-1}\right\|_{1} \leqslant(2+\sigma) \sigma\left(2\|\mathrm{y}\|_{1}\|\mathrm{x}\|_{1}+\|\overline{\mathrm{y}}\|_{1}\|x\|_{1}+\|\bar{x}\|_{1}\|y\|_{1}\right)+\sigma\left(\|y\|_{1}+\|\bar{y}\|_{1}\right) .
$$

Proof. Rewrite the inverse formula for $\mathrm{T}_{\mathrm{C}}$ as

$$
\mathrm{T}_{\mathrm{C}}^{-1}=\left(\mathrm{L}_{\mathrm{y}}+\mathrm{R}_{\overline{\mathrm{y}}}\right) \mathrm{R}_{\mathrm{x}}^{(1)} \mathrm{D}-\left(\mathrm{L}_{\mathrm{x}}+\mathrm{R}_{\overline{\mathrm{x}}}\right) \mathrm{R}_{\mathrm{y}}^{(0)} \mathrm{D},
$$

where

$$
\begin{aligned}
& \mathrm{L}_{\mathrm{y}}=\left(\begin{array}{cccc}
\mathrm{y}_{1} & & & \\
\mathrm{y}_{2} & \mathrm{y}_{1} & & \\
\vdots & \ddots & \ddots & \\
\mathrm{y}_{\mathrm{n}} & \cdots & \mathrm{y}_{2} & \mathrm{y}_{1}
\end{array}\right) \\
& \mathrm{R}_{\overline{\mathrm{y}}}=\left(\begin{array}{cccc}
0 & i y_{n} & \cdots & i y_{2} \\
& 0 & \ddots & \vdots \\
& & \ddots & i y_{n} \\
& & & 0
\end{array}\right), \\
& \mathrm{L}_{x}=\left(\begin{array}{cccc}
x_{1} & & & \\
x_{2} & x_{1} & & \\
\vdots & \ddots & \ddots & \\
x_{n} & \cdots & x_{2} & x_{1}
\end{array}\right) \text {, } \\
& \mathrm{R}_{\overline{\mathrm{x}}}=\left(\begin{array}{cccc}
0 & \mathfrak{i} x_{\mathrm{n}} & \cdots & i x_{2} \\
& 0 & \ddots & \vdots \\
& & \ddots & i x_{n} \\
& & & 0
\end{array}\right) \text {, } \\
& \mathrm{R}_{x}^{(1)}=\left(\begin{array}{cccc}
1 & x_{n} & \cdots & x_{2} \\
& 1 & \ddots & \vdots \\
& & \ddots & x_{n} \\
& & & 1
\end{array}\right) \text {, } \\
& R_{y}^{(0)}=\left(\begin{array}{ccccc}
0 & y_{n} & y_{n-1} & \cdots & y_{2} \\
& 0 & y_{n} & \ddots & \vdots \\
& & \ddots & \ddots & y_{n-1} \\
& & & \ddots & y_{n} \\
& & & & 0
\end{array}\right)
\end{aligned}
$$

Thus

$$
\begin{aligned}
\left\|T_{C}^{-1}-\hat{T}_{C}^{-1}\right\|_{1} & \leqslant\left\|L_{y} R_{x}^{(1)}-\hat{L}_{y} \hat{R}_{x}^{(1)}\right\|_{1}+\left\|R_{\bar{y}} R_{x}^{(1)}-\hat{R}_{\bar{y}} \hat{R}_{x}^{(1)}\right\|_{1}+\left\|L_{x} R_{y}^{(0)}-\hat{L}_{x} \hat{R}_{y}^{(0)}\right\|_{1}+\left\|R_{\bar{x}} R_{y}^{(0)}-\hat{R}_{\bar{x}} \hat{R}_{y}^{(0)}\right\|_{1} \\
& \triangleq \tau_{1}+\tau_{2}+\tau_{3}+\tau_{4} .
\end{aligned}
$$

For the first term $\tau_{1}$, we have,

$$
\begin{aligned}
\tau_{1} & \leqslant\left\|L_{y} R_{x}^{(1)}-\hat{L}_{y} \hat{R}_{x}^{(1)}\right\|_{1} \\
& \leqslant\left\|L_{y} R_{x}^{(1)}-\hat{L}_{y} R_{x}^{(1)}+\hat{L}_{y} R_{x}^{(1)}-\hat{L}_{y} \hat{R}_{x}^{(1)}\right\|_{1} \\
& \leqslant\left\|L_{y} R_{x}^{(1)}-\hat{L}_{y} R_{x}^{(1)}\right\|_{1}+\left\|\hat{L}_{y} R_{x}^{(1)}-\hat{L}_{y} \hat{R}_{x}^{(1)}\right\|_{1}
\end{aligned}
$$




$$
\begin{aligned}
& \leqslant\left\|\mathrm{L}_{y}-\hat{\mathrm{L}}_{y}\right\|_{1}\left\|\mathrm{R}_{x}^{(1)}\right\|_{1}+\left\|\hat{\mathrm{L}}_{y}\right\|_{1}\left\|\mathrm{R}_{x}^{(1)}-\hat{\mathrm{R}}_{x}^{(1)}\right\|_{1} \\
& \leqslant\|y-\hat{y}\|_{1}\left(1+\|x\|_{1}\right)+(1+\sigma)\|y\|_{1} \sigma\|x\|_{1} \\
& \leqslant \sigma\|y\|_{1}\left(1+\|x\|_{1}\right)+(1+\sigma)\|y\|_{1} \sigma\|x\|_{1} \\
& \leqslant(2+\sigma) \sigma\|y\|_{1}\|x\|_{1}+\sigma\|y\|_{1} .
\end{aligned}
$$

Then $\tau_{2}, \tau_{3}, \tau_{4}$ can be derived similarly, which are

$$
\tau_{2} \leqslant(2+\sigma) \sigma\|\bar{y}\|_{1}\|x\|_{1}+\sigma\|\bar{y}\|_{1}, \quad \tau_{3} \leqslant(2+\sigma) \sigma\|y\|_{1}\|x\|_{1}, \quad \tau_{4} \leqslant(2+\sigma) \sigma\|y\|_{1}\|\bar{x}\|_{1} .
$$

We obtain the designed result by summing the above four inequalities.

Remark 4.2. Under the assumptions and notations of Theorem 4.1, it can be obtained the same upper bound of $\left\|\mathrm{T}_{\mathrm{C}}^{-1}-\hat{\mathrm{T}}_{\mathrm{C}}^{-1}\right\|_{\infty}$ with the 1-norm in a similar way,

$$
\left\|\mathrm{T}_{\mathrm{C}}^{-1}-\hat{\mathrm{T}}_{\mathrm{C}}^{-1}\right\|_{\infty} \leqslant(2+\sigma) \sigma\left(2\|y\|_{1}\|x\|_{1}+\|\bar{y}\|_{1}\|x\|_{1}+\|\bar{x}\|_{1}\|y\|_{1}\right)+\sigma\left(\|y\|_{1}+\|\bar{y}\|_{1}\right) .
$$

So we are in a position to present the upper bound with respect to the 2-norm, since

$$
\left\|\mathrm{T}_{\mathrm{C}}^{-1}-\hat{\mathrm{T}}_{\mathrm{C}}^{-1}\right\|_{2}^{2} \leqslant\left\|\mathrm{~T}_{\mathrm{C}}^{-1}-\hat{\mathrm{T}}_{\mathrm{C}}^{-1}\right\|_{1}\left\|\mathrm{~T}_{\mathrm{C}}^{-1}-\hat{\mathrm{T}}_{\mathrm{C}}^{-1}\right\|_{\infty},
$$

and we have from (4.1) and (4.2) that

$$
\begin{aligned}
\left\|\mathrm{T}_{\mathrm{C}}^{-1}-\hat{\mathrm{T}}_{\mathrm{C}}^{-1}\right\|_{2} \leqslant & (2+\sigma) \sigma \sqrt{\mathfrak{n}(\mathfrak{n}-1)}\left(\|\bar{y}\|_{2}\|x\|_{2}+\|\bar{x}\|_{2}\|y\|_{2}\right) \\
& +2(2+\sigma) \sigma n\|y\|_{2}\|x\|_{2}+\sigma \sqrt{\mathfrak{n}}\left(\|\bar{y}\|_{2}+\|y\|_{2}\right),
\end{aligned}
$$

as $\|x\|_{1} \leqslant \sqrt{n}\|x\|_{2},\|\bar{x}\|_{1} \leqslant \sqrt{n-1}\|\bar{x}\|_{2},\|y\|_{1} \leqslant \sqrt{n}\|y\|_{2}$, and $\|\bar{y}\|_{1} \leqslant \sqrt{n-1}\|\bar{y}\|_{2}$. Therefore, the formula presented in Theorem 2.2 is forward stable.

Theorem 4.3. Let $\mathrm{H}_{\mathrm{C}}$ be an $\mathrm{n} \times \mathrm{n}$ conjugate-Hankel matrix and well conditioned. Let $\sigma>0$ and let $\hat{\xi}, \hat{\eta}, \hat{\bar{\xi}}, \hat{\bar{\eta}}$ be the corresponding numerical least squares solutions of the linear systems for deriving the formula (3.1). Denote by $\hat{\mathrm{H}}_{\mathrm{C}}^{-1}$ the inverse of $\hat{\mathrm{H}}_{\mathrm{C}}$. If $\frac{\|\hat{\xi}-\xi\|_{1}}{\|\varepsilon\|_{1}} \leqslant \sigma, \frac{\|\hat{\eta}-\eta\|_{1}}{\|\eta \eta\|_{1}} \leqslant \sigma, \frac{\|\hat{\bar{\xi}}-\bar{\xi}\|_{1}}{\|\bar{\xi}\|_{1}} \leqslant \sigma, \frac{\|\hat{\bar{\eta}}-\bar{\eta}\|_{1}}{\|\bar{\eta}\|_{1}} \leqslant \sigma$, then

$$
\left\|\mathrm{H}_{\mathrm{C}}^{-1}-\hat{\mathrm{H}}_{\mathrm{C}}^{-1}\right\|_{1} \leqslant(2+\sigma) \sigma\left(2\|\eta\|_{1}\|\xi\|_{1}+\|\bar{\eta}\|_{1}\|\xi\|_{1}+\|\bar{\xi}\|_{1}\|\eta\|_{1}\right)+\sigma\left(\|\bar{\eta}\|_{1}+\|\eta\|_{1}\right) .
$$

Proof. Rewrite the inverse formula for $\mathrm{H}_{\mathrm{C}}$ as

$$
H_{C}^{-1}=\left(L_{\eta}+R_{\bar{\eta}}\right) R_{\xi}^{(1)} \mathbb{D}-\left(L_{\xi}+R_{\bar{\xi}}\right) R_{\eta}^{(0)} \mathbb{D}
$$

where

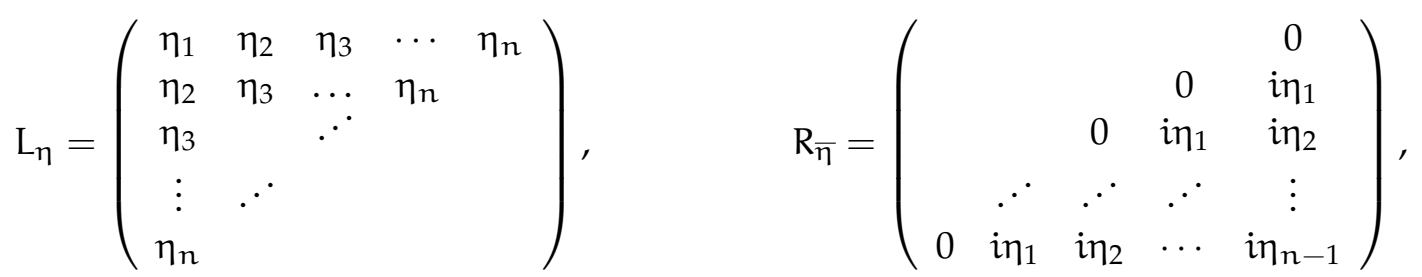

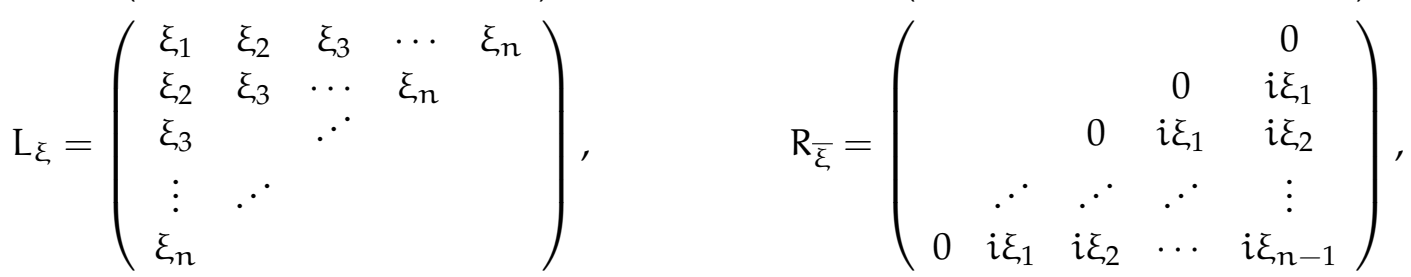




$$
\mathrm{R}_{\xi}^{(1)}=\left(\begin{array}{cccc}
1 & \xi_{1} & \cdots & \xi_{n-1} \\
& 1 & \ddots & \vdots \\
& & \ddots & \xi_{1} \\
& & & 1
\end{array}\right), \quad R_{\eta}^{(0)}=\left(\begin{array}{ccccc}
0 & \eta_{1} & \eta_{2} & \cdots & \eta_{n-1} \\
& 0 & \eta_{1} & \ddots & \vdots \\
& & 0 & \ddots & \eta_{2} \\
& & & \ddots & \eta_{1} \\
& & & & 0
\end{array}\right)
$$

We obtain

$$
\begin{aligned}
\left\|H_{C}^{-1}-\hat{H}_{C}^{-1}\right\|_{1} & \leqslant\left\|L_{\eta} R_{\xi}^{(1)}-\hat{L}_{\eta} \hat{R}_{\xi}^{(1)}\right\|_{1}+\left\|R_{\bar{\eta}} R_{\xi}^{(1)}-\hat{R}_{\bar{\eta}} \hat{R}_{\xi}^{(1)}\right\|_{1}+\left\|L_{\xi} R_{\eta}^{(0)}-\hat{L}_{\xi} \hat{R}_{\eta}^{(0)}\right\|_{1}+\left\|R_{\bar{\xi}} R_{\eta}^{(0)}-\hat{R}_{\bar{\xi}} \hat{R}_{\eta}^{(0)}\right\|_{1} \\
& \triangleq \kappa_{1}+\kappa_{2}+\kappa_{3}+\kappa_{4} .
\end{aligned}
$$

For the first term $\kappa_{1}$, we have,

$$
\kappa_{1} \leqslant(2+\sigma) \sigma\|\eta\|_{1}\|\xi\|_{1}+\sigma\|\eta\|_{1} .
$$

Then $\kappa_{2}, \kappa_{3}, \kappa_{4}$ can be derived similarly, which are

$$
\kappa_{2} \leqslant(2+\sigma) \sigma\|\bar{\eta}\|_{1}\|\xi\|_{1}+\sigma\|\bar{\eta}\|_{1}, \quad \kappa_{3} \leqslant(2+\sigma) \sigma\|\eta\|_{1}\|\xi\|_{1}, \quad \kappa_{4} \leqslant(2+\sigma) \sigma\|\eta\|_{1}\|\bar{\xi}\|_{1} .
$$

We obtain the desired result by summing the above four inequalities.

Remark 4.4. Under the assumptions and notations of Theorem 4.3, it can be obtained the similar upper bound for $\left\|\mathrm{H}_{\mathrm{C}}^{-1}-\hat{\mathrm{H}}_{\mathrm{C}}^{-1}\right\|_{\infty}$ :

$$
\left\|H_{C}^{-1}-\hat{H}_{C}^{-1}\right\|_{\infty} \leqslant(2+\sigma) \sigma\left(2\|\eta\|_{1}\|\xi\|_{1}+\|\bar{\eta}\|_{1}\|\xi\|_{1}+\|\bar{\xi}\|_{1}\|\eta\|_{1}\right)+\sigma\left(\|\bar{\eta}\|_{1}+\|\eta\|_{1}\right) .
$$

So we are in a position to give the upper bound with respect to the 2-norm, since

$$
\left\|\mathrm{H}_{\mathrm{C}}^{-1}-\hat{\mathrm{H}}_{\mathrm{C}}^{-1}\right\|_{2}^{2} \leqslant\left\|\mathrm{H}_{\mathrm{C}}^{-1}-\hat{\mathrm{H}}_{\mathrm{C}}^{-1}\right\|_{1}\left\|\mathrm{H}_{\mathrm{C}}^{-1}-\hat{\mathrm{H}}_{\mathrm{C}}^{-1}\right\|_{\infty},
$$

and we have from (4.3) and (4.4) that

$$
\begin{aligned}
\left\|H_{C}^{-1}-\hat{H}_{C}^{-1}\right\|_{2} \leqslant & (2+\sigma) \sigma \sqrt{n(n-1)}\left(\|\bar{\eta}\|_{2}\|\xi\|_{2}+\|\bar{\xi}\|_{2}\|\eta\|_{2}\right) \\
& +2(2+\sigma) \sigma n\|\eta\|_{2}\|\xi\|_{2}+\sigma \sqrt{n}\left(\|\bar{\eta}\|_{2}+\|\eta\|_{2}\right),
\end{aligned}
$$

as $\|\xi\|_{1} \leqslant \sqrt{\mathfrak{n}}\|\xi\|_{2},\|\bar{\xi}\|_{1} \leqslant \sqrt{\mathfrak{n}-1}\|\bar{\xi}\|_{2},\|\eta\|_{1} \leqslant \sqrt{\mathfrak{n}}\|\eta\|_{2}$, and $\|\bar{\eta}\|_{1} \leqslant \sqrt{\mathfrak{n}-1}\|\bar{\eta}\|_{2}$. Therefore, the formula presented in Theorem 3.2 is forward stable.

\section{Two algorithms on finding $\mathrm{T}_{\mathrm{C}}^{-1}$ and $\mathrm{H}_{\mathrm{C}}^{-1}$}

In this section, two algorithms on finding $\mathrm{T}_{\mathrm{C}}^{-1}$ and $\mathrm{H}_{\mathrm{C}}^{-1}$ are given.

Algorithm 5.1. Using Theorem 2.2, we proceed with

Step 1. Compute $v=\left(\begin{array}{lllll}0 & c^{0}\left(t_{1-n}-i t_{1}\right) & \cdots & c^{n-3}\left(t_{-2}-i t_{n-2}\right) & c^{n-2}\left(t_{-1}-i t_{n-1}\right.\end{array}\right)^{\top}$.

Step 2. Compute $x=\left(\begin{array}{lllll}x_{1} & x_{2} & \ldots & x_{n}\end{array}\right)^{\top}$ and $y=\left(\begin{array}{llll}y_{1} & y_{2} & \ldots & y_{n}\end{array}\right)^{\top}$ by solving the systems of equations

$$
\mathrm{T}_{\mathrm{C}} \mathrm{x}=v \text { and } \mathrm{T}_{\mathrm{C}} \mathrm{y}=\mathrm{e}_{1} \text {. }
$$

Step 3. Compute $\mathrm{T}_{\mathrm{C}}^{-1}$ via formula (2.2).

Algorithm 5.2. Using Theorem 3.2, we proceed with

Step 1. Compute $\hat{v}=\left(\begin{array}{lllll}0 & c^{0}\left(h_{0}-i h_{n}\right) & c\left(h_{1}-i h_{n+1}\right) & \cdots & c^{n-2}\left(h_{n-2}-i h_{2 n-2}\right)\end{array}\right)^{\top}$.

Step 2. Compute $\xi=\left(\begin{array}{llll}\xi_{1} & \xi_{2} & \cdots & \xi_{n}\end{array}\right)^{\top}$ and $\eta=\left(\begin{array}{llll}\eta_{1} & \eta_{2} & \cdots & \eta_{n}\end{array}\right)^{\top}$ by solving the systems of equations

$$
\mathrm{H}_{\mathrm{C}} \xi=\hat{v} \text { and } \mathrm{H}_{\mathrm{C}} \eta=e_{1} .
$$

Step 3. Compute $\mathrm{H}_{\mathrm{C}}^{-1}$ via formula (3.1). 


\section{Numerical example}

In this section we give two examples to demonstrate our main results.

Example 6.1. Give a $4 \times 4$ CT matrix

$$
\mathrm{T}_{C}=\left(\begin{array}{cccc}
i & 2 i & 3 i & 4 i \\
5 i & -i & -2 i & -3 i \\
i & -5 i & i & 2 i \\
3 i & -i & 5 i & -i
\end{array}\right)
$$

It is obvious that $T_{C}$ is invertible. By Algorithm 5.1, we have

Step 1. Compute $v$ by Lemma 2.1:

$$
v=\left(\begin{array}{c}
0 \\
-5+4 i \\
-1-3 i \\
-3+2 i
\end{array}\right)
$$

Step 2. Compute $x, y$ by the systems of equations $T_{C} x=v, T_{C} y=e_{1}$ :

$$
x=\left(\begin{array}{c}
\frac{6462+8768 i}{10000} \\
\frac{3685-3236 i}{10000} \\
\frac{307+177 i}{10000} \\
\frac{-4603-1851 i}{10000}
\end{array}\right), y=\left(\begin{array}{c}
-\frac{1229}{10000} i \\
-\frac{951}{10000} i \\
\frac{177}{10000} i \\
-\frac{1850}{10000} i
\end{array}\right)
$$

Step 3. Compute $\mathrm{T}_{\mathrm{C}}^{-1}$ by using the equation (2.2):

$$
\begin{aligned}
& \mathrm{T}_{\mathrm{C}}^{-1}=\left(\begin{array}{cccc}
-\frac{1229}{10000} i & \frac{1850}{10000} & -\frac{177}{10000} & \frac{951}{10000} \\
-\frac{951}{10000} i & -\frac{1229}{10000} i & \frac{1850}{10000} & -\frac{177}{10000} \\
\frac{177}{10000} i & -\frac{951}{10000} i & -\frac{1229}{10000} i & \frac{1850}{10000} \\
-\frac{1050}{10000} i & \frac{177}{10000} i & -\frac{951}{10000} i & -\frac{1229}{10000} i
\end{array}\right) \\
& \times\left(\begin{array}{cccc}
1 & \frac{4603+1851 i}{10000} & \frac{307+177 i}{10000} & \frac{-3685+3236 i}{1000} \\
0 & -1 & -\frac{4603+1851 i}{10000} & -\frac{307+177 i}{1000} \\
0 & 0 & 1 & \frac{4603+1851 i}{10000} \\
0 & 0 & 0 & -1
\end{array}\right) \\
& +\left(\begin{array}{cccc}
\frac{6462+8768 i}{1000} & \frac{-4603 i+1851}{10000} & \frac{307 i-177}{10000} & \frac{3685 i+3236}{10000} \\
\frac{3685-3236 i}{10000} & \frac{6462+8768 i}{1000} & \frac{-4603 i+1851}{10000} & \frac{307 i-177}{10000} \\
\frac{307+177 i}{10000} & \frac{3685-3236 i}{10000} & \frac{6462+8868 i}{10000} & \frac{-4603 i+1851}{100000} \\
\frac{-4603-1851 i}{10000} & \frac{307+177 i}{10000} & \frac{365-3236 i}{10000} & \frac{6462+8688 i}{10000}
\end{array}\right) \\
& \times\left(\begin{array}{cccc}
0 & -\frac{1850}{10000} i & -\frac{177}{10000} i & -\frac{951}{10000} i \\
0 & 0 & \frac{1850}{10000} i & \frac{1770}{1000} i \\
0 & 0 & 0 & -\frac{1850}{10000} i \\
0 & 0 & 0 & 0
\end{array}\right) \\
& =\left(\begin{array}{cccc}
-\frac{1229}{10000} i & -\frac{1761}{10000} i & -\frac{152}{10000} i & \frac{63}{10000} i \\
-\frac{951}{10000} i & -\frac{229}{10000} i & \frac{1635}{10000} i & \frac{152}{10000} i \\
\frac{177}{10000} i & \frac{976}{10000} i & \frac{229}{10000} i & -\frac{1762}{10000} i \\
-\frac{1850}{10000} i & -\frac{177}{10000} i & -\frac{951}{10000} i & \frac{1229}{10000} i
\end{array}\right) .
\end{aligned}
$$


Example 6.2. Give a $4 \times 4 \mathrm{CH}$ matrix

$$
H_{C}=\left(\begin{array}{cccc}
i & i & 2 i & 3 i \\
-i & -2 i & -3 i & -4 i \\
2 i & 3 i & 4 i & 5 i \\
-3 i & -4 i & -5 i & -2 i
\end{array}\right)
$$

It is obvious that $\mathrm{H}_{\mathrm{C}}$ is invertible. By Algorithm 5.2, we have

Step 1. Compute $\hat{v}$ by Lemma 3.1:

$$
\hat{v}=\left(\begin{array}{c}
0 \\
4+i \\
-5-i \\
2+2 i
\end{array}\right)
$$

Step 2. Compute $\xi, \eta$ by the systems of equations $H_{C} \xi=\hat{v}, H_{C} \eta=e_{1}$ :

$$
\xi=\left(\begin{array}{c}
1-3 i \\
-\frac{3}{4}+6 i \\
-\frac{1}{2}-3 i \\
\frac{1}{4}+i
\end{array}\right), \eta=\left(\begin{array}{c}
-i \\
2 i \\
-i \\
0
\end{array}\right) .
$$

Step 3. Compute $\mathrm{H}_{\mathrm{C}}^{-1}$ by using the equation (3.1):

$$
\begin{aligned}
\mathrm{H}_{\mathrm{C}}^{-1}= & \left(\begin{array}{cccc}
-\mathfrak{i} & 2 i & -\mathfrak{i} & 0 \\
2 i & -\mathfrak{i} & 0 & 1 \\
-\mathfrak{i} & 0 & 1 & -2 \\
0 & 1 & -2 & 1
\end{array}\right)\left(\begin{array}{cccc}
1 & -1+3 i & -\frac{3}{4}+6 i & \frac{1}{2}+3 i \\
0 & -1 & 1-3 i & \frac{3}{4}-6 i \\
0 & 0 & 1 & -1+3 i \\
0 & 0 & 0 & -1
\end{array}\right) \\
& +\left(\begin{array}{cccc}
1-3 i & -\frac{3}{4}+6 i & -\frac{1}{2}-3 i & \frac{1}{4}+\mathfrak{i} \\
-\frac{3}{4}+6 i & -\frac{1}{2}-3 i & \frac{1}{4}+i & 3+i \\
-\frac{1}{2}-3 i & \frac{1}{4}+i & 3+i & -6-\frac{3}{4} \mathfrak{i} \\
\frac{1}{4}+\mathfrak{i} & 3+i & -6-\frac{3}{4} \mathfrak{i} & 3-\frac{1}{2} \mathfrak{i}
\end{array}\right)\left(\begin{array}{cccc}
0 & -i & -2 i & -i \\
0 & 0 & i & 2 i \\
0 & 0 & 0 & -i \\
0 & 0 & 0 & 0
\end{array}\right) \\
= & \left(\begin{array}{cccc}
-\mathfrak{i} & -2 i & -\mathfrak{i} & 0 \\
2 i & -\frac{1}{4} \mathfrak{i} & -\frac{3}{2} \mathfrak{i} & -\frac{1}{4} \mathfrak{i} \\
-\mathfrak{i} & \frac{3}{2} \mathfrak{i} & 2 \mathfrak{i} & \frac{1}{2} \mathfrak{i} \\
0 & -\frac{1}{4} \mathfrak{i} & -\frac{1}{2} \mathfrak{i} & -\frac{1}{4} \mathfrak{i}
\end{array}\right) .
\end{aligned}
$$

\section{Implications of application for decomposition formulas (2.2) and (3.1)}

We now propose how the analogue of formulas (2.2) and (3.1) lead to a more efficient way to calculate $\mathrm{T}_{\mathrm{C}}^{-1} \mathrm{~b}$ and $\mathrm{H}_{\mathrm{C}}^{-1} \mathrm{~b}$.

Ammar and Gader [1] proposed that the circulant-vector product $z=C_{x} y$ is equal to the cyclic convolution of the vectors $x$ and $y$, which we denote by $x * y$. Moreover, $z=x * y$ if and only if $F_{n} z=$ $\left(F_{n} x\right) \cdot\left(F_{n} y\right)$, where $x \cdot y$ denotes the componentwise product of $x$ and $y, n F_{n}=\left[\omega_{n}^{-j k}\right]_{j, k=0}^{n-1}$ and $\omega_{n}=$ $e^{2 \pi i / n}$ denotes the principal $n$th root of unity. Consequently, $z=W_{n}\left(\left(F_{n} x\right) \cdot\left(F_{n} y\right)\right)$, where $W_{n}=F_{n}^{-1}=$ $\left[\omega_{n}^{j k}\right]_{0}^{n-1}=n \bar{F}_{n}$. So $z$ can be computed in $\tau(n)+O(n)$ arithmetic operations, where $\tau(n)$ denotes the amount of computation required to perform one real FFT of order $n$.

According to computational implications of Ammar and Gader [1], we know that the computation of $x=T_{C}^{-1} b$ using our formula (2.2) requires at most $9 \tau(n)+O(n)$ computations, as well as the computation of $x=\mathrm{H}_{\mathrm{C}}^{-1} \mathrm{~b}$ using our formula (3.1) requires at most $9 \tau(n)+\mathrm{O}(\mathrm{n})$ computations, too. 
In Table 1, we list the number of real arithmetic operations (additions and multiplications) required by the algorithms of Gohberg-Semencul formula-like [12] and the Gohberg-Semencul formula [17] for $T_{C}^{-1} b$ and $\mathrm{H}_{\mathrm{C}}^{-1} \mathrm{~b}$, as well as operation counts for the implementations of formula (2.2), (3.1) described above.

The value of our formulas (2.2), (3.1) increase dramatically in situations in which $\mathrm{T}_{\mathrm{C}}^{-1} \mathrm{~b}_{\mathrm{k}}$ and $\mathrm{H}_{\mathrm{C}}^{-1} \mathrm{~b}_{\mathrm{k}}$ are to be obtained for several different vectors $b_{k}$. Instances of this situation are in the iterative improvement of solutions.

Table 1: Operation counts.

\begin{tabular}{l|c}
\hline \hline \multicolumn{1}{c|}{ Algorithm } & Number of real arithmetic operations \\
\hline \hline 1. The Gohberg-Semencul formula-like for $T_{C}$ & $42 n \log _{2} n[12]$ \\
2. The Gohberg-Semencul formula-like for $\mathrm{H}_{C}$ & $42 n \log _{2} n[12]$ \\
3. The Gohberg-Semencul formula for $T_{C}$ & $28 n \log _{2} n$ [17] \\
4. The Gohberg-Semencul formula for $\mathrm{H}_{C}$ & $28 n \log _{2} n[17]$ \\
\hline 5. The imaginary circulant GS formula (2.2) for $\mathrm{T}_{C}$ & $18 n \log _{2} n$ \\
6. The imaginary left circulant GS formula (3.1) for $\mathrm{H}_{C}$ & $18 n \log _{2} n$ \\
\hline
\end{tabular}

\section{Acknowledgment}

This work was supported by the GRRC program of Gyeonggi Province [(GRRC SUWON 2016-B3), Development of cloud Computing-based Intelligent Video Security Surveillance System with Active Tracking Technology]. Their support is gratefully acknowledged

\section{References}

[1] G. Ammar, P. Gader, A variant of the Gohberg-Semencul formula involving circulant matrices, SIAM J. Matrix Anal. Appl., 12 (1991), 534-540. 1, 7

[2] G. S. Ammar, W. B. Gragg, The generalized Schur algorithm for the superfast solution of Toeplitz systems, Rational approximation and applications in mathematics and physics, Łańcut, (1985), Lecture Notes in Math., Springer, Berlin, 1237 (1987), 315-330. 1

[3] G. S. Ammar, W. B. Gragg, Superfast solution of real positive definite Toeplitz systems, SIAM Conference on Linear Algebra in Signals, Systems, and Control, Boston, Mass., (1986), SIAM J. Matrix Anal. Appl., 9 (1988), 61-76. 1

[4] S. Barnett, M. J. C. Gover, Some extensions of Hankel and Toeplitz matrices, Linear and Multilinear Algebra, 14 (1983), 45-65. 1

[5] M. I. Español, M. E. Kilmer, Multilevel approach for signal restoration problems with Toeplitz matrices, SIAM J. Sci. Comput., 32 (2010), 299-319. 1

[6] I. C. Gohberg, A. A. Semencul, The inversion of finite Toeplitz matrices and their continual analogues, (Russian) Mat. Issled., 7 (1972), 201-233. 1

[7] M. J. C. Gover, S. Barnett, Generating polynomials for matrices with Toeplitz or conjugate-Toeplitz inverses, Linear Algebra Appl., 61 (1984), 253-275. 1

[8] M. J. C. Gover, S. Barnett, Inversion of certain extensions of Toeplitz matrices, J. Math. Anal. Appl., 100 (1984), $339-353$. 1

[9] M. J. C. Gover, S. Barnett, Characterisation and properties of r-Toeplitz matrices, J. Math. Anal. Appl., 123 (1987), 297-305. 1

[10] M. H. Gutknecht, M. Hochbruck, The stability of inversion formulas for Toeplitz matrices, Special issue honoring Miroslav Fiedler and Vlastimil Pták, Linear Algebra Appl., 223/224 (1995), 307-324. 1

[11] J. R. Jain, An efficient algorithm for a large Toeplitz set of linear equations, IEEE Trans. Acoust. Speech Signal Process., 27 (1979), 612-615. 1

[12] Z.-L. Jiang, J.-X. Chen, The explicit inverse of nonsingular conjugate-Toeplitz and conjugate-Hankel matrices, J. Appl. Math. Comput., 53 (2017), 1-16. 1, 7, 1

[13] Z.-L. Jiang, X.-T. Chen, J.-M. Wang, The explicit inverses of CUPL-Toeplitz and CUPL-Hankel matrices, East Asian J. Appl. Math., 7 (2017), 38-54. 1

[14] X.-Y. Jiang, K.-C. Hong, Equalities and inequalities for norms of block imaginary circulant operator matrices, Abstr. Appl. Anal., 2015 (2015), 5 pages. 1, 2.2

[15] X.-Y. Jiang, K.-C. Hong, Skew cyclic displacements and inversions of two innovative patterned Matrices, Appl. Math. Comput., 308 (2017), 174-184. 1 
[16] Z.-L. Jiang, Y.-C. Qiao, S.-D. Wang, Norm equalities and inequalities for three circulant operator matrices, Acta Math. Sin. (Engl. Ser.), 33 (2017), 571-590. 2.2

[17] Z.-L. Jiang, T.-Y. Tam, Y.-F. Wang, Inversion of conjugate-Toeplitz matrices and conjugate-Hankel matrices, Linear Multilinear Algebra, 65 (2017), 256-268. 1, 7, 1

[18] Z.-L. Jiang, D.-D. Wang, Explicit group inverse of an innovative patterned matrix, Appl. Math. Comput., 274 (2016), 220-228. 1

[19] Z.-L. Jiang, H.-X. Xin, H.-W. Wang, On computing of positive integer powers for r-circulant matrices, Appl. Math. Comput., 265 (2015), 409-413. 2.2

[20] Z.-L. Jiang, T.-T. Xu, Norm estimates of $\omega$-circulant operator matrices and isomorphic operators for $\omega$-circulant algebra, Sci. China Math., 59 (2016), 351-366.

[21] Z.-L. Jiang, Z.-X. Zhou, Circulant matrices, Chengdu Technology University Publishing Company, Chengdu, (1999). $1,2.2,3.2$

[22] N. T. Keliba, D. Huylebrouck, A note on conjugate Toeplitz matrices, Linear Algebra Appl., 139 (1990), 103-109. 1

[23] L. Lerer, M. Tismenetsky, Generalized Bezoutian and the inversion problem for block matrices, I, General scheme, Integral Equations Operator Theory, 9 (1986), 790-819. 1

[24] X.-G. Lv, T.-Z. Huang, A note on inversion of Toeplitz matrices, Appl. Math. Lett., 20 (2007), 1189-1193. 1

[25] J.-S. Mei, R.-Q. Wang, Eigenvalue analysis of circulant matrices extended from the Toeplitz matrices, Prog. Geophys., 28 (2013), 265-269. 1, 2.2

[26] M. K. Ng, Iterative methods for Toeplitz systems, Numerical Mathematics and Scientific Computation, Oxford University Press, New York, (2004). 1

[27] M. K. Ng, J.-Y. Pan, Weighted Toeplitz regularized least squares computation for image restoration, SIAM J. Sci. Comput., 36 (2014), B94-B121. 1

[28] F. J. Simois, J. I. Acha, A new algorithm for real data convolutions with j-circulants, IEEE Signal Process. Lett., 18 (2011), 655-658. 1

[29] W. F. Trench, An algorithm for the inversion of finite Toeplitz matrices, J. Soc. Indust. Appl. Math., 12 (1964), 515-522. 1

[30] Y.-W. Wen, M. K. Ng, W.-K. Ching, H. Liu, A note on the stability of Toeplitz matrix inversion formulas, Appl. Math. Lett., 17 (2004), 903-907. 1

[31] P.-P. Xie, Y.-M. Wei, The stability of formula of the Gohberg-Semencul-Trench type for Moore-Penrose and group inverses of Toeplitz matrices, Linear Algebra Appl., 498 (2016), 117-135. 1 